\title{
Second-strand Library Sequencing
}

National Cancer Institute

\section{Source}

National Cancer Institute. Second-strand Library Sequencing. NCI Thesaurus. Code C150426.

Directional sequencing, where the first read of the read pair (or in case of single end reads, the only read) is from the transcript strand. 